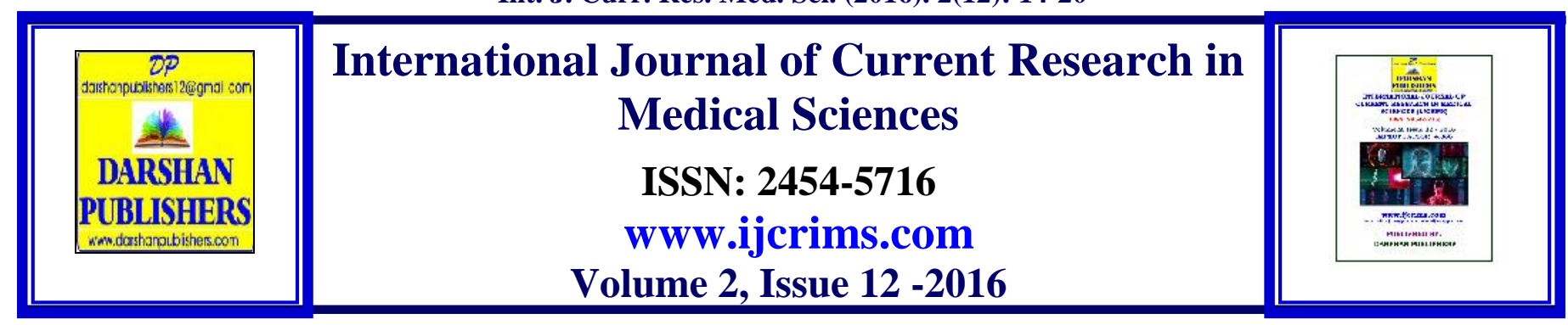

\title{
The study on the expression of sulfotransferases2A1 in uterine Leiomyoma and its clinical significance
}

\author{
Pankaj Pratap Deo ${ }^{1 *}$, Wang Min ${ }^{1,}$ Sabbu Shah ${ }^{2}$, Dinesh Prasad Yadav ${ }^{3}$ \\ Suneel Piryani ${ }^{4}$ \\ ${ }^{1}$ Department of Obstetrics and Gynecology, Janaki Medical College \& TH Janakpur \\ ${ }^{1}$ Department of Obstetrics and Gynecology, Shengjing Hospital, China Medical \\ University,Liaoning Shenyang 110004,China. \\ 2 Department of Radiology, Janaki Medical College \& TH Janakpur. \\ ${ }^{3}$ Department of Biochemistry, Janaki Medical College, Janakpur \\ ${ }^{4}$ Department of Community Medicine and Public Health, Institute of Medicine, Tribhuvan University, \\ Kathmandu, Nepal. \\ *Corresponding author: Dr. Pankaj Pratap Deo \\ Assistant Professor, Department of Obstetrics and Gynecology, Janaki Medical College \& TH Janakpur, \\ Nepal. Mob: +977-9851130833 e-mail: pankajpratapdeo@gmail.com
}

\begin{abstract}
Aims: The objective of this study was to examine the expression of Sulfotransferases2A1 in Uterine Leiomyoma and to determine its Clinical Significance using Western Blot method.

Methods: A hospital based cross sectional study was conducted in Department of Obstetrics and Gynecology, Shengjing Hospital, China Medical University from December 2013 to June 2014. Patients included were of age 36 45 years, in proliferative phase of menstrual cycle, with no history of diabetes, hyperthyroidism, ovarian cancer, other comorbidities or hormonal therapy for last 3 months. Tissue samples were taken from fibroid tissue and extra capsular myometrium in 30 uterine leiomyomatous patients and from normal myometrium in 10 uterine prolapse cases. Western Blot assay was used to detect SULT2A1 expression in uterine leiomyoma tissue, uterine leiomyoma adjacent tissue and normal myometrial tissue.

Results: The expression of SULT2A1 was seen in 12 (40\%) specimens of fibroid tissue, 17 (56.6\%) specimens of extra capsular myometrium and $6(60 \%)$ specimens of normal myometrium. Significant difference was seen between specimens of fibroid tissue and extra capsular myometrium ( $\mathrm{p}$-value $<0.05$ ), fibroid tissue and normal myometrium ( $\mathrm{p}$-value $<0.05$ ) but not between extra capsular myometrium and normal myometrium ( $\mathrm{p}$-value $>0.05$ ).

Conclusion: This study gives evidence of SULT2A1 expression in uterine fibroid, fibroid extra capsular tissue and normal myometrium. The higher level of SULT2A1 protein expression in the normal myometrium compared to extra capsular tissue or uterine fibroid tissue, points to its possible role in the tumor genesis. This provides a promising theoretical basis for advent of non-surgical treatment of uterine fibroid through specific protein expression targeting.
\end{abstract}

Keywords: Uterine fibroid; SULT2A1; Western Blot 


\section{Introduction}

At present, uterine leiomyoma stands as a major health problem worldwide. It is the most common uterine tumor in women of reproductive age group. Approximately $70-80 \%$ of women of reproductive age are afflicted with this disease [1, 2]. More than $60 \%$ of the laparotomies done for pelvic diseases are attributed to uterine leiomyoma (ULMs) [2]. Besides, it accounts for various morbidities like abdominal pain, dysmenorrhea, menorrhagia, infertility [1, 3, 4]. In addition, it is the commonest indication for hysterectomy in most of the countries and necessitates treatment cost hiking over billions annually $[1,3,5]$.

Despite the high prevalence rate and health disturbances, exact pathogenesis still remains to be elucidated. A number of literatures describe the risk factors associated to fibroid and its tumor genesis. Early menarche, nulliparity, increasing age, obesity, diet including red meat and ham, and certain race and geography favor its occurrence. However, menopause, smoking and exercise prevent its growth. The role of OCP and HRT still remain inconclusive [6]. It is evident that ULMs are highly dependent on ovarian steroids. Recent studies have shown increased expression of estrogen receptor $(E R \alpha)$, aberrant micro RNA, HSD17B1 rs605059 gene polymorphism associated with ULMs [7, 8, 9, 10, 11]. Literature reports steroid sulfotransferase (SULT) as major enzyme catalyzing sulfation of steroids, drugs and xenobiotic. SULT2A1 is the major isoform that catalyzes sulfation of various steroids including DHEA to DHEAS, the precursors of both androgen and estrogen [12, 13,14]. Abnormal SULT1A1 and SULT1E1 gene expression have been indicated in leiomyoma and endometrial Cancer [15]. Also, estrogen sulfotransferase (EST) is known to be related to progression and metastasis of estrogen dependent breast cancer [16]. Although the enzymatic activity of SULT2A1 has been studied in some detail, little is known about its expression in any tissue. Therefore, this study is an attempt to explore SULT2A1 expression in uterine leiomyoma and to determine its clinical significance.

\section{Materials and Methods}

A cross sectional study was conducted in the Department of Obstetrics and Gynecology, Shengjing Hospital of China Medical University from December 2013 to June 2014.

Specimens were collected from 30 uterine leiomyoma patients after total or subtotal hysterectomy and 10 uterine prolapse patients (not with uterine fibroids, adenomyosis or endometrial hyperplasia) after total hysterectomy. The inclusion criteria were a) patient's age 36 - 45 years, b) proliferative phase of menstrual cycle, c) not under any hormonal therapy for last 3 months and d) with no history of diabetes, hyperthyroidism, ovarian cancer and other comorbidities. In uterine leiomyoma specimen, tissue sample was taken each $1 \mathrm{~cm}$ deep from the fibroid tissue and extra capsular myometrium (adjacent to fibroid capsule). In uterine prolapse specimen, tissue sample was taken from normal myometrium. Western Blot assay was used to detect SULT2A1 expression in uterine leiomyoma tissue, uterine leiomyoma adjacent tissue and normal myometrial tissue.

Statistical analysis was done using SPSS 17.0 software. ANOVA comparative analysis and Student-Newman-Keuls (SNK) pair wise comparison were done to test the difference of expression of SLUT2A1 between the different tissues. P-value $<0.05$ indicated that the analysis was statistically significant and $\mathrm{P}<0.01$ indicating that it was very significant. The study was approved by the hospital ethical committee. Informed written consent was collected from the patients.

\section{Experimental reagents and instruments used}

1. SULT2A1 antibody of rabbit anti-human polyclonal from Beijing Biosynthesis Biotechnology Co., Ltd.

2. Secondary antibody of donkey anti-human polyclonal from British Abcam.

3. BCA protein assay kit from Beijing Cell Chip Biotechnology Co., Ltd.

4. PVDF membrane (0.45um) from America Millipore. 
5. SDS-PAGE protein sample buffer $(5 x)$ from Shanghai Beyotime Biotechnology Co., Ltd.

6. Western Blot kit from Shanghai Beyotime Biotechnology Co., Ltd.

7. Western Blot with a protein marker from Lithuania Fermentas.

8. Constant temperature table: ZHWY-211C from Shanghai Yiheng technology.

9. Gel Doc 1000 gel imaging systems from the United States BIO-RAD company.

10. Electric heater water bath Model HHSY11-Ni produced from Beijing Changfeng Instruments Corporation.

\section{Experimental Method}

\section{Western Blot -detection of protein expression}

\section{(A) Sample preparation}

(1) Selection of sample: The specimens were removed from freezer $\left(-80{ }^{\circ} \mathrm{C}\right)$ and checked one by one for any damage. Then $0.1 \mathrm{~g}$ sample was taken from each specimen, washed twice with cold PBS and put into fresh EP tube.

(2) Configuration of Cell lysate: RIPA Lysis buffer $(750 \mathrm{ml})$ was added to each EP tube and mixed well. PMSF was also added making final concentration $1 \mathrm{mM}$.Then cell culture was centrifuged in a microcentrifuge $\left(4^{\circ} \mathrm{C}, 12000 \mathrm{rpm}\right.$, $15 \mathrm{~min})$. The tubes were gently removed from the centrifuge. The supernatant was aspirated and put in fresh tube, separately and saved at $\left(-80^{\circ} \mathrm{C}\right)$.

(3) Configuration of tissue lysate: Tissues were dissected in ultrasonic cell disruptor and placed in EP tubes. These were then immersed in liquid nitrogen, lysis buffer was added and homogenized with an electric homogenizer, then centrifuged in a micro centrifuge $\left(4^{\circ} \mathrm{C}, 12000 \mathrm{rpm}, 15 \mathrm{~min}\right)$. The tubes were gently removed from the centrifuge. The protein containing supernatant was aspirated and put in fresh tube, separately and saved at (-80 $\left.{ }^{\circ} \mathrm{C}\right)$.

(4) Protein quantization

(a) Working solution was prepared according to the standard solution and number of samples. $B C A$ reagent $A$ : reagent $B=50: 1$ formulation, an appropriate amount of BCA working solution was made. BCA working solution was stable at room temperature within 24 hours.

(b)Dilution of standard solution with PBS buffer was done to give final concentration of $0.5 \mathrm{mg} / \mathrm{ml}$ and standard curve was prepared for the fluid.

(c) 151 PBS buffer and 51 sample were added to the sample bottle and repeated three times.

(d) $2001 \mathrm{BCA}$ working solution was added to each well at $37^{\circ} \mathrm{C}$ and left for 30 minutes.

(e) It was then cooled down to room temperature. Using Elisa A562, the protein concentration was determined according to the standard curve.

(5) After boiling the protein samples and the sample buffer mix $\left(100{ }^{\circ} \mathrm{C}\right.$ for 5 min), these were instantly then placed on ice and cooled to room temperature.

\section{(B) Electrophoresis, Transfer of proteins and staining (Western Blotting)}

\section{(1) Preparation of PAGE Gel}

The proteins were separated, according to their molecular weight, on polyacrylamide gel using standard technique SDS-polyacrylamide agarose gel electrophoresis (SDS-PAGE).

Table 1: Showing the Gel formulation

\begin{tabular}{lllllll}
\hline Gel & $\begin{array}{l}\mathrm{H} 2 \mathrm{O} \\
(\mathrm{ml})\end{array}$ & $\begin{array}{l}30 \% \\
\text { Acrylamid } \\
((\mathrm{ml})\end{array}$ & $\begin{array}{l}1.5 \mathrm{M} \text { Tris } \\
(\mathrm{ml})\end{array}$ & $\begin{array}{l}10 \% \text { SDS } \\
(\mathrm{ml})\end{array}$ & $\begin{array}{l}10 \% \text { APS } \\
(\mathrm{ml})\end{array}$ & $\begin{array}{l}\text { TEMED } \\
(\mathrm{ml})\end{array}$ \\
\hline $\begin{array}{l}\text { 10\%Separatin } \\
\text { g gel 5ml }\end{array}$ & 1.3 & 1.7 & $\begin{array}{l}(\mathrm{pH} 8.8) \\
1.9\end{array}$ & 0.05 & 0.05 & 0.002 \\
$\begin{array}{l}\text { 5\%Stacking } \\
\text { gel 2ml }\end{array}$ & 1.4 & 0.33 & $\begin{array}{l}(\mathrm{pH} 6.8) \\
0.25\end{array}$ & 0.02 & 0.02 & 0.002 \\
\hline
\end{tabular}


(2) The samples were mixed with 41 of prestained protein marker on the Marker track.

(3) Electrophoresis was done by setting the voltage at $80 \mathrm{~V}$ and adjusting the voltage to $120 \mathrm{~V}$ until marker bands were seen separated into the separating gel. Electrophoresis was continued for about two hours.

(4) After electrophoresis, the gel was removed and placed in transfer buffer. PVDF membrane and filter were cut to appropriate size depending on the gel size. PVDF membrane was pretreated by immersing it in methanol for $1 \mathrm{~min}$. The membrane was then put with filter paper into the transfer buffer.

(5) The gel and membrane were sandwiched between filter paper and cotton pads (three layers of filter paper - gel - PVDF membrane - triple filter paper) and finally covered with cotton pad. Ensuring that there were no air bubbles between gel and membrane, all were finally clamped in electric transfer folder.

(6) The clip was put into the transfer slot, to make the clip black-face to face slot black-face and clip white face to face slot red face. A piece of ice was placed at the side of the slot to cool when the transfer produced heat.

(7) Blocking: 5\% skimmed milk was configured with TBS-T as blocking buffer. The PVDF membrane was rinsed once with TBS-T for $5 \mathrm{~min}$. The film was incubated for 2 hours at $4^{0} \mathrm{C}$ under agitation.

(8)Primary antibody incubation: Post dilution, the antibody was incubated overnight at $4^{\circ} \mathrm{C}$ with membrane in a hybridization bag, sealed air tight after removing air bubbles.

(9) Secondary antibody incubation: The membrane was then rinsed in TBS-T thrice, 10 min per wash, to remove residual primary antibody. The appropriate secondary antibody was selected and diluted in blocking buffer. It was incubated with membrane, at room temperature for 2 hours in a hybridization bag, sealed air tight after removing air bubbles.

(10) Development: After secondary antibody incubation, it was rinsed with TBS-T thrice, 10 min per wash. Then ECL + detection kit was used and film was developed in a darkroom. Lastly, photographs were taken to save the picture.

\section{Results}

In specimens from 30 uterine leiomyoma patients, the expression of SULT2A1 was seen in $12(40 \%)$ specimens of fibroid tissue and 17 (56.6\%) specimens of fibroid extracapsular myometrium. Whereas, in 10 uterine prolapse patients, the expression of SULT2A1 was seen in $6(60 \%)$ specimens. (Table 2)

Significant difference was seen in expression of SLUT2A1 between specimens of uterine fibroid tissue and fibroids extra capsular myometrium ( $\mathrm{p}$ value $<0.05$ ), uterine fibroid tissue and normal myometrium ( $p$-value $<0.05$ ). However, there was no significant difference in expression of SLUT2A1 between fibroids extra capsular myometrium and normal myometrium (p-value $>0.05)$.

ANOVA comparative analysis of SULT2A1 protein content in the three types of tissues showed significant variance $(\mathrm{P}<0.001)$

Student-Newman-Keuls (SNK) pair wise comparisons showed difference between each groups.

Table 2: SULT2A1 protein relative content $\left({ }^{\bar{x}} \pm s\right)$ analysis results

\begin{tabular}{|c|c|c|c|}
\hline Specimen & Frequency & Percent & SULT2A1 (Mean \pm SD) \\
\hline Uterine fibroids & 12 & $40.00 \%$ & $0.207 \pm 0.058$ \\
\hline Extra capsular tissue & 17 & $56.67 \%$ & $0.332 \pm 0.108$ \\
\hline Normal myometrium & 6 & $60.00 \%$ & $0.532 \pm 0.108$ \\
\hline
\end{tabular}


Figure 1: Pattern of expression of SULT2A1 in uterine leiomyoma, myoma extra capsular myometrium and normal myometrium tissue

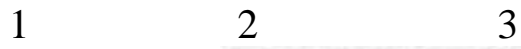

SULT2A1

3

GAPDH

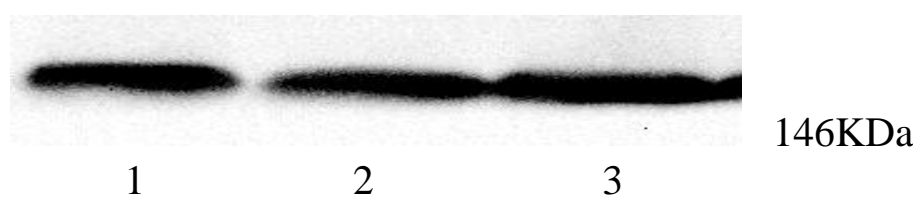

Figure 1: Western Blot method to detect the expression of SULT2A1.

1: SULT2A1 expression in uterine fibroids tissue

2: SULT2A1 expression in the extra capsular tissue of uterine fibroids

3: SULT2A1 expression in normal myometrium tissue

\section{Discussion}

Recent studies found that uterine fibroids, endometrial cancer and other gynecological tumors, abnormal expression of SULT1E1 and SULT1A1, and found SULT1E1 and SULT1A1 polymorphism associated with gynecologic cancer susceptibility.

SULT1A1 catalyze the sulfate conjugation of phenolic compounds including steroid hormones, catecholamines and phenolic drugs, and also participate in the bio activation of procarcinogens.

SULT1E1 strongest affinity of estrogenin all the SULTs [8], and it's the most closely relationship with physiological metabolism of estrogen [9]. As an important detoxification enzymes, catalytic estrogens (estrone and 17 - estradiol) and its metabolites (such as catechol estrogens) sulfate metabolism. It can form the inactive form of estrogen also is estrogen sulfate compound, estrogen sulfate.

Prof Wang Ming use chemiluminescent method for determination of content of E2 and progesterone and testosterone in uterine leiomyoma patients and healthy women's serum and tissue homogenates, and immunohistochemical staining SULT1E1 protein expression. Result show that $[11,12]$ :In the uterine fibroid tissue,E2 were significantly higher than in leiomyoma extra capsular tissue ,In the uterine fibroid tissue,SULT1E1 protein expression levels were significantly lower than in leiomyoma extra capsular tissue .Therefore infer the occurrence of uterine myoma was related to the decrease of SULT1E1 enzyme activity. Its mechanism may be:SULT1E1 catalyzed endogenous estrogen sulfating reaction, formation of estrogen inactive form - sulfating estrogen, this compound cannot be combined with estrogen receptor and lost activity, and it can reduce circulation and target tissue estrogen exposure levels.SULT1E1 lower expression in uterine fibroids organizations can lead to elevated levels of endogenous estrogen, estrogen has raised PR content effect, PR was positively related to the intensity of ER protein expression, higher content of ER alpha indirectly, promote the growth of uterine fibroids.

Hydroxysteroid sulfotransferase SULT2A1 catalyzes the sulfation of hydroxysteroids. It plays an important role in the detoxification of hydroxyl-containing xenobiotics and in the regulation of the biological activities of hydroxysteroids. ERR $\alpha$ is an orphan member of the nuclear receptor superfamily that is closely related to estrogen receptor alpha (ER $\alpha)$. Here we report that the mRNA expression of human SULT2A1 was suppressed by ERR $\alpha$ in myometrium cells. To investigate the mechanisms of this regulation, the effects of ERR $\alpha$ on human 
SULT2A1 promoter transcription in myoma cells were investigated. Reporter luciferase assay results showed that $\mathrm{ERR} \alpha$ significantly represses human SULT2A1 promoter transcription in myoma cells. Deletion analysis indicated that human SULT2A1 promoter region between positions -188 and -130 is necessary for its repression by ERR $\alpha$ in myoma cells. The 5' DNA -188 to -130 region of human SULT2A1 contains IR2 and DR4 hormone response elements and two putative ERR $\alpha$ response elements (ERREs) (ERRE188: GCAAGCTCA and ERRE155: ATAAGTTCA). Our results suggest that ERR $\alpha$ may play an important role in promoting the leiomyoma and in regulating endogenous hydroxysteroid activities via the regulation of SULT2A1. Sulfuric acid transferase enzyme members, SULT2A1 uterine fibroids, may play an important role in the development.

Human SULT2A1 has been mapped to chromosome 19q13.3 (Luu-The et al. 1995, Otterness et al. 1995).

SULT2A1 is located on chromosome 19:48373723bp-48389654bp, molecular weight: $35 \mathrm{kDa}$. SULT2A1 in the liver, colon, kidney, testicle, lymph nodes, cerebral cortex and adrenal tissue were expressed, especially in colorectal cancer, liver cancer, pancreatic cancer, stomach cancer, bladder cancer has a high expression [17]. There are studies that RORs can activate the SULT2A1 promoter, and the expression of SULT2A1 is positively correlated with the expression of RORs, which indicates that it can regulate the expression of SULT2A1.. It is also reported that vitamin $\mathrm{D}$ receptor (VDR) can stimulate the expression of endogenous SULT2A1. Estrogen receptor associated receptor ERR can down regulate the expression of SULT2A1.

In summary, SULT gene family associated with uterine fibroids, endometrial cancer and other gynecological tumor's development, the mechanism may be estrogen SULT abnormal, made topical estrogen synthesis and metabolism, increased target organ estrogen exposure.SULT2A1, as a member of the SULT2 family, whose main catalytic DHEA, pregnelone and dehydroepiandrosterone ketone, hydroxy steroid sulfation, like SULT1E1, SULT1A1, SULT1A3, SULT2A1 also catalyze the metabolism of estrogen. SULT2A1 relationship between estrogen metabolism and suggest it occurs in uterine fibroids, may play an important role in development.

SULT2A1 protein expression in uterine leiomyoma and clinical significance.

SULT2A1 protein in uterine fibroids, fibroids extracapsular muscle tissue and normal myometrium were expressed. SULT2A1 protein relative content in uterine fibroids was $0.207 \pm$ 0.058, SULT2A1 protein relative content in fibroids extracapsular tissue was $0.332 \pm 0.108$, SULT2A1 protein relative content in normal myometrium tissue was $0.532 \pm 0.108$. SULT2A1 protein relative content comparative analysis of variance $F$ value is $28.379, P<0.001$ in three different tissues, there is a significant difference. Student-Newman-Keuls (SNK) pairwise comparisons showed that, between any two groups are not the same numbers.

\section{Conclusion}

The existing high prevalence rate of leiomyoma, associated morbidities and the financial burden of hysterectomies necessitate that alternative therapies are developed. This target can be attained only with better understanding of leiomyoma at cellular level.

This study provides evidence of SULT2A1 protein expression in uterine fibroid, fibroid extra capsular tissue and normal myometrium. The expression level of SULT2A1 protein in the normal myometrium was significantly higher than in leiomyoma extra capsular tissue or uterine fibroid tissue, inferring that this protein may have a role in the pathogenesis and development of uterine fibroid. Its expression pattern shows that the mechanism may be inhibiting factor in the incidence of uterine fibroids. This finding provides promising theoretical basis for advent of non-surgical treatment of uterine fibroid through specific protein expression targeting. 


\section{Acknowledgements}

Facilities provided by the Department of Obstetrics and Gynecology, Shengjing Hospital are highly acknowledged. The authors are thankful to hospital management and staffs of Shengjing Hospital, China for their consistent support and cooperation during this research.

\section{References}

1. Kim et al. The role of progesterone signaling in the pathogenesis of uterine leiomyoma. Mol Cell Endo.2012 jul25; 358(2):223-31.

2. Pradhan et al. Uterine Myoma-A Profile of Nepalese women. Nepal J Obstet. Gynaecol 2006 Nov-Dec; 1(2):47-50.

3. Asada et al. Potential link between estrogen receptor- $\alpha$ gene hypomethylation and uterine fibroid formation. Mol Hum Repro.2008; 14(9):539-45

4. Khan et al. Uterine fibroids: current perspectives. Int. J Women's Health. 2014Jan; 6:95-114

5. Stewart et al. Uterine fibroid. Lancet. 2001Jan; 357:293-8.

6. Flake et al. Etiology and Pathogenesis of Uterine Leiomyomas. Environ Health Perspect. 2003; 111(8):1037-54.

7. Luo et al. The Expression and Potential Regulatory Function of MicroRNAs in the Pathogenesis of Leiomyoma. Semin Repro Med. 2008Nov; 26(6): 500-14.

8. Zavadil et al. Profiling and Functional Analyses of MicroRNAs and Their Target Gene Products in Human Uterine Leiomyomas. PLoS ONE. 2010; 5(8):e12362.

9. Danielson et al. A Differentiation-Based MicroRNA Signature Identifies

How to cite this article:

Pankaj Pratap Deo, Wang Min' Sabbu Shah, Dinesh Prasad Yadav, Suneel Piryani. (2016). The study on the expression of sulfotransferases2A1 in uterine Leiomyoma and its clinical significance. Int. J. Curr. Res. Med. Sci. 2(12): 14-20.

DOI: http://dx.doi.org/10.22192/ijcrms.2016.02.12.003
Leiomyosarcoma as a Mesenchymal Stem Cell - Related Malignancy. AMJ2010; 177:908-17.

10. Chuang et al. miR-200c is aberrantly expressed in leiomyomas in an ethnicdependent manner and targets ZEBs, VEGFA, TIMP2, and FBLN5. Endocrine -Related Cancer. 2012; 19:541-56.

11. Cong et al. Polymorphism in genes HSD17B1 and HSD17B2 and uterine leiomyoma risk in Chinese women. Arch Gynae Obstet. 2012; 286:701-5.

12. Duffel et al. Substrate inhibition in human hydroxysteroid sulfotranferase SULT2A1: Studies on the formation of catalytically nonproductive enzyme compexes. Arch Bioch and Biophy.2011;507:232-40.

13. Sinclair et al. Molecular cloning and regulation of porcine SULT2A1:relationship between SULT2A1 expression and sulfoconjugation of androsterone. J Mol Endo. 2006; 36 (2):301-11.

14. Lu et al. Identification and Characterization of Two Amino Acids Critical for the Substrate Inhibition of Human Dehydroepiandrosterone Sulfotransferase (SULT2A1). Mol Pharmacol. 2008;73(3):660-68.

15. Rui et al. SULT Family and Gynaecological Tumors. J Int Obstet Gynae.2011;38(4):32427.

16. Tanaka et al. Estrogen sulfotransferase and sulfatase: Roles in the regulation of estrogen activity in human uterine endometrial carcinomas. Cancer Sci 2003; 94:871-76.

17. Flynn M, Jamison M, Datta S, Myers E. Health care resource use for uterine fibroid tumors in the United States. Am J Obstet Gynecol. 2006; 195:955-64.

\begin{tabular}{|c|c|}
\hline \multicolumn{2}{|c|}{ Access this Article in Online } \\
\hline \multirow{2}{*}{ 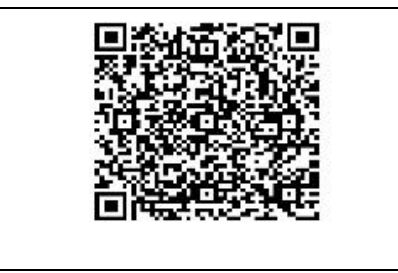 } & $\begin{array}{l}\text { Website: } \\
\text { www.ijcrims.com }\end{array}$ \\
\hline & $\begin{array}{l}\text { Subject: } \\
\text { Medical Sciences }\end{array}$ \\
\hline Quick Response Code & \\
\hline
\end{tabular}

